\title{
Achievable Rates of Compress-and-Forward Cooperative Relaying on Gaussian Vector Channels
}

\author{
Sebastien Simoens, Olga Muñoz ${ }^{\dagger}$ and Josep Vidal ${ }^{\dagger}$ \\ Motorola Labs, Paris, France. E-mail : simoens@motorola.com \\ "Universitat Politècnica de Catalunya (UPC), Signal Processing and Communications group, Barcelona, Spain
}

\begin{abstract}
The Compress-and-Forward (C\&F) cooperative relaying strategy is known to outperform Decode-and-Forward (D\&F) when the relay is close to the destination. In this paper, we derive achievable rates on Gaussian vector channels with cooperative $C \& F$ relaying. In order to extend previous information-theoretic results from the scalar to the vector Gaussian channel, we exploit recent results in distributed source coding. Like in source coding with side information at the decoder, the relay applies a Conditional Karhunen Loeve Transform (CKLT) to its observed signal, followed by a separate Wyner-Ziv encoding of each output stream with a different rate and under a sum-rate constraint. However, these Wyner-Ziv coding rates are such that the total mutual information between the source and destination is maximized. This differs from the conventional source coding approach in which the rates are selected to minimize the total squared distortion, leading to the well-known reverse-waterfilling algorithm. We show that the maximization of the $C \& F$ mutual information is also a convex problem. The optimum Wyner-Ziv coding rates have a simple analytical expression, and can be obtained by a waterfilling algorithm. Finally, we illustrate these results by simulations of MIMO-OFDM relaying in a system similar to IEEE802.16.
\end{abstract}

Index Terms-cooperative, relaying, compress, forward

\section{INTRODUCTION}

In recent wireless standardization efforts, such as IEEE $802.11 \mathrm{n}$ and $802.16 \mathrm{e}$, the supported physical layer is able to operate close to capacity by combining the benefits of Coded Orthogonal Frequency Division Multiplexing (COFDM), multiple antenna transmission, powerful error-correcting codes (e.g. LDPC) and Adaptive Modulation and Coding (see e.g. [1] for an illustration in $802.11 \mathrm{n}$ context). Moreover, the addition of relaying features is also being discussed in $802.11 \mathrm{~s}$ and $802.16 \mathrm{j}$ Task Groups, mostly at the MAC but also at the PHY level. This highlights the need for relay channel capacity bounds on Gaussian vector channels.

This paper assumes a single relay for simplification. Moreover, the relay is assumed to operate in half-duplex mode, because this greatly simplifies practical implementation. We assume Time Division Duplex (TDD) operation in the rest of this paper, which allows us to divide the time into two slots where the relay receives during first slot of duration $t$ and transmits during second slot of duration $1-t$ with $0 \leq t \leq 1$. We focus on cooperative relaying (see e.g. [3] for an overview), in which the destination combines the signals received from the source and the relay.

The relay may be either regenerative or non-regenerative. An example of regenerative relaying strategy is D\&F, in which the relay decodes all the information sent by the source before forwarding it to the destination. Non-regenerative relaying schemes include the Amplify-and-Forward (A\&F) and C\&F strategies. An A\&F relay simply retransmits the signal received from the source after amplification. In [4] and [5] the A\&F concept is extended to vector channels by allowing the relay to perform a linear processing on the received signal before transmitting it to the destination. The $\mathrm{C} \& \mathrm{~F}$ approach was initially suggested in [10]. A C\&F relay quantizes the signal observed during $1^{\text {st }}$ slot and sends a compressed version of this signal in $2^{\text {nd }}$ slot. The compression exploits the fact that the observations at the relay and destination during slot 1 are correlated. More precisely, the relay employs source coding with side information at the destination (a.k.a. Wyner-Ziv coding [9]). Recent work [2] shows that the achievable rate of $\mathrm{D} \& \mathrm{~F}$ is higher when the relay is close to the source, but C\&F approaches the max-flow min-cut upper-bound on capacity as the relay gets closer to the destination, while A\&F capacity is severely limited by the time sharing parameter constrained to $t=1 / 2$. Note that A\&F can however become attractive in systems where aggressive reuse of the $2^{\text {nd }}$ slot is possible [16].

In [6], Høst-Madsen and Zhang derive achievable rates for the TDD C\&F scalar Gaussian relay channel. They show that with the $C \& F$ relaying strategy, the mutual information between the source and the destination depends on a "compression noise" which differs from the distortion in general. This compression noise was first introduced by Wyner [9], who derived the rate-distortion function for source coding with Gaussian source and Gaussian side information. In [8], we attempted to derive achievable C\&F rates in Gaussian vector channel. However, we assumed that the distortion was equal to the compression noise, which is valid only in certain conditions clarified later in this paper. Besides, we also assumed that side information was available at the relay and destination, which prevented us from stating whether the rates were achievable or not. In [7], Gastpar et al. studied 
distributed source coding and showed that the rate-distortion coding of a Gaussian vector source with side information at the decoder could be achieved by first applying a Conditional Karhunen-Loeve Transform (CKLT) to the source and then separately performing Wyner-Ziv encoding of each output stream. Gastpar focused on minimizing the total squared distortion. The rate at which each eigenmode is quantized is univocally related to the distortion on this eigenmode. The solution which minimizes total squared distortion is given by the so-called reverse-waterfilling algorithm [12] which attempts to spread the distortion uniformly over the eigenvalues of the conditional covariance matrix of the relay observation. In this paper, we build upon [9] and [7] to generalize the notion of "compression noise" to Gaussian vector channels in section II. Then in section III we derive the strategy which maximizes the achievable $\mathrm{C} \& \mathrm{~F}$ rate on Gaussian vector channels. We show that the simple analytical solution to this convex problem has a waterfilling interpretation, and consists in granting more bits to the eigenmodes which are the largest contributors to $C \& F$ mutual information. Finally, in section IV we illustrate the gain provided by the maximum mutual information strategy compared to the minimum total distortion strategy by simulations in a MIMO-OFDM system similar to IEEE802.16. We also benchmark the performance of $\mathrm{C} \& \mathrm{~F}$ with other strategies such as D\&F and A\&F.

\section{WYNER-ZIV CODING OF GAUSSIAN VECTOR SOURCE}

\section{A. Signal Model}

We consider a Source S, a Relay R and a destination D. For simplification, we perform calculations assuming that only $\mathrm{R}$ transmits during the $2^{\text {nd }}$ slot, but at the end of section III we will discuss how the results should be modified to account for simultaneous transmission of $\mathrm{S}$ and $\mathrm{R}$ during the $2^{\text {nd }}$ slot. The signals received at $\mathrm{R}$ and $\mathrm{D}$ during the $1^{\text {st }}$ slot are denoted by:

$$
\mathbf{y}_{S-R}=\mathbf{H}_{S-R} \mathbf{x}_{S}+\mathbf{n}_{R} \text { and } \mathbf{y}_{S-D}=\mathbf{H}_{S-D} \mathbf{x}_{S}+\mathbf{n}_{D, 1} \text { (1) }
$$

where $\mathbf{H}_{S-R}$ and $\mathbf{H}_{S-D}$ are channel matrices of respective dimensions $N_{R} \times N_{S}$ and $N_{D} \times N_{S}$. In the following, we will denote the covariance matrix of a vector $\mathbf{x}$ by $\mathbf{R}_{\mathbf{x}}$. In equation (1), vectors $\mathbf{n}_{R}$ and $\mathbf{n}_{D, 1}$ are assumed complex and gaussian of respective covariance matrices $\mathbf{R}_{\mathbf{n}_{R}}$ and $\mathbf{R}_{\mathbf{n}_{D}}$. The source transmit power is bounded by $P_{S}$. Likewise, during the $2^{\text {nd }}$ slot, the destination receives

$$
\mathbf{y}_{R-D}=\mathbf{H}_{R-D} \mathbf{x}_{R}+\mathbf{n}_{D, 2}
$$

where $\operatorname{tr}\left(\mathbf{R}_{\mathbf{x}_{R}}\right) \leq P_{R}$ and $\mathbf{n}_{D, 2} \sim \mathbb{C} \mathcal{N}\left(0, \mathbf{R}_{\mathbf{n}_{D}}\right)$

The vectors $\mathbf{y}_{S-R}$ and $\mathbf{y}_{S-D}$ are complex Gaussian with respective covariance matrices :

$$
\begin{gathered}
\mathbf{R}_{\mathbf{y}_{S-R}}=\mathbf{H}_{S-R} \mathbf{R}_{\mathbf{x}_{s}} \mathbf{H}_{S-R}^{H}+\mathbf{R}_{\mathbf{n}_{R}} \\
\mathbf{R}_{\mathbf{y}_{S-D}}=\mathbf{H}_{S-D} \mathbf{R}_{\mathbf{x}_{s}} \mathbf{H}_{S-D}^{H}+\mathbf{R}_{\mathbf{n}_{D, 1}}
\end{gathered}
$$

Their cross-correlation matrix equals:

$$
\mathbf{R}_{\mathbf{y}_{S-D}, \mathbf{y}_{S-R}}=\mathbf{H}_{S-D} \mathbf{R}_{\mathbf{x}_{s}} \mathbf{H}_{S-R}^{H}
$$

During the $2^{\text {nd }}$ phase, the relay sends a compressed version of its observations $\mathbf{y}_{S-R}$ to the destination, which reconstructs an estimate $\hat{\mathbf{y}}_{S-R}$ using side information $\mathbf{y}_{S-D}$. The mutual information between the relay and destination is:

$$
I\left(\mathbf{x}_{R} ; \mathbf{y}_{D}\right)=\log \left|\mathbf{I}_{N_{R}}+\mathbf{H}_{R-D} \mathbf{R}_{\mathbf{x}_{R}} \mathbf{H}_{R-D}^{H} \mathbf{R}_{\mathbf{n}_{D}}^{-1}\right|
$$

The average rate at which the relay can encode its observation is constrained to be lower than the average mutual information between $\mathrm{R}$ and D. Each vector $\mathbf{y}_{S-R}$ can thus be encoded on $R(t)$ bits with:

$$
R(t) \leq \frac{1-t}{t} I\left(\mathbf{x}_{R} ; \mathbf{y}_{D}\right)
$$

This rate limitation is going to give rise to distortion. In the following we denote by $D \hat{=}\left\|\mathbf{y}_{S-R}-\hat{\mathbf{y}}_{S-R}\right\|^{2}$ the total squared distortion. In the next sections, we investigate the ratedistortion trade-off and the impact on the mutual information of the cooperative $C \& F$ scheme.

\section{B. Source coding of Gaussian vectors with side information at the decoder}

The characterization of the rate-distortion function for Gaussian scalar sources with Gaussian scalar side information at the decoder is performed by Wyner in [9]. In [6], HøstMadsen and Zhang show that the mutual information of cooperative $\mathrm{C} \& \mathrm{~F}$ relaying depends on a "compression noise" introduced in Wyner's landmark paper. The compression noise significantly differs from the distortion at low signal to distortion ratio. More recently, Gastpar et al. compute in [7] the rate-distortion function of Gaussian vector sources. In this section we build upon their work but further detail the coding scheme by defining a vector compression noise, which is used in the next section to compute the $\mathrm{C} \& \mathrm{~F}$ mutual information.

Let first define the Conditional Karhunen-Loeve Transform (CKLT) as in [7]. Given the knowledge of $\mathbf{y}_{S-D}$, the vector $\mathbf{y}_{S-R}$ is Gaussian distributed [11] of mean

$$
E\left[\mathbf{y}_{S-R} \mid \mathbf{y}_{S-D}\right]=\mathbf{R}_{\mathbf{y}_{S-R}, \mathbf{y}_{S-D}} \mathbf{R}_{\mathbf{y}_{S-D}}^{-1} \mathbf{y}_{S-D}
$$

and covariance matrix denoted by $\mathbf{R}_{\mathbf{y}_{S-R} \mid \mathbf{y}_{S-D}}$ and equal to:

$$
\mathbf{R}_{\mathbf{y}_{S-R} \mid \mathbf{y}_{S-D}}=\mathbf{R}_{\mathbf{y}_{S-R}}-\mathbf{R}_{\mathbf{y}_{S-R}, \mathbf{y}_{S-D}} \mathbf{R}_{\mathbf{y}_{S-D}^{-1}}^{-R_{\mathbf{y}_{S-D}, \mathbf{y}_{S-R}}}
$$

The CKLT is defined as the matrix $\mathbf{U}^{H}$ such that:

$$
\mathbf{R}_{\mathbf{y}_{s-l} \mid \mathbf{y}_{s-p}}=\mathbf{U} \operatorname{diag}(\mathbf{s}) \mathbf{U}^{H}
$$

The columns of $\mathbf{U}$ are the eigenvectors of the conditional covariance matrix, and the vector $\mathbf{S}$ contains the associated eigenvalues. We denote by $N_{\text {eig }}$ the number of non-zero eigenvalues.

Having defined the CKLT, we can now introduce the notations used in the two coding schemes of Fig. 1 and Fig. 2. 
On these figures, $\boldsymbol{\psi}$ is a "compression noise" vector of i.i.d. components of variance $\eta_{i}$, i.e. $\psi_{i} \sim \mathbb{C} \mathcal{N}\left(0, \eta_{i}\right)$ with

$$
\eta_{i}=\frac{s_{i} d_{i}}{s_{i}-d_{i}} \text { for } 1 \leq i \leq N_{e i g}
$$

We also define matrix $\mathbf{A}=\operatorname{diag}(\mathbf{a})$ with $a_{i}=\left(s_{i}-d_{i}\right) / s_{i}$. These two schemes are equivalent, in the sense that they result in the same relation between the source $\mathbf{y}_{S-R}$ and the reconstructed signal $\hat{\mathbf{y}}_{S-R}$, which is given by:

$$
\hat{\mathbf{y}}_{S-R}=\mathbf{U A U} \mathbf{U}^{H} \mathbf{y}_{S-R}+\mathbf{U A} \boldsymbol{\psi}+\mathbf{U K} \mathbf{y}_{S-D}
$$

where $\mathbf{K} \hat{=}(\mathbf{I}-\mathbf{A}) \mathbf{U}^{H} \mathbf{R}_{\mathbf{y}_{S-R}, \mathbf{y}_{S-D}} \mathbf{R}_{\mathbf{y}_{S-D}}^{-1}$

Before going into further considerations on the theoretical aspects, we can try to provide an intuitive understanding of the figures as follows: the coding scheme of Fig. 1 assumes that side information $\mathbf{y}_{S-D}$ is available at both the source and the decoder. The encoder first removes the conditional expectation $E\left[\mathbf{y}_{S-R} \mid \mathbf{y}_{S-D}\right]$ from the source $\mathbf{y}_{S-R}$, and performs ratedistortion coding of the remainder. The decoder adds back the conditional expectation to the reconstructed signal in order to obtain $\hat{\mathbf{y}}_{S-R}$. The scheme on Fig. 2 only assumes side information at the decoder, which is the framework of this paper.

Moreover, it can be checked that the $i$ th component $d_{i}$ of vector $\mathbf{d}$ corresponds to the distortion on the $i$ th component of the CKLT output $\mathbf{U}^{H} \mathbf{y}_{S-R}$. Indeed, introducing (8) into (12) yields:

$\mathbf{U}^{H}\left(\hat{\mathbf{y}}_{S-R}-\mathbf{y}_{S-R}\right)=(\mathbf{A}-\mathbf{I}) \mathbf{U}^{H}\left(\mathbf{y}_{S-R}-E\left[\mathbf{y}_{S-R} \mid \mathbf{y}_{S-D}\right]\right)+\mathbf{A} \boldsymbol{\psi}$

and from the definition of conditional covariance:

$E\left[\left\|\mathbf{y}_{S-R}-\hat{\mathbf{y}}_{S-R}\right\|^{2} \mid \mathbf{y}_{S-D}\right]=\sum_{i=1}^{N_{\text {eig }}}\left(a_{i}-1\right)^{2} s_{i}+a_{i}^{2} \eta_{i}=\sum_{i=1}^{N_{\text {eig }}} d_{i}$

Denoting by $D \triangleq \sum_{i=1}^{N_{\text {eig }}} d_{i}$ the total squared distortion, we can now state the following result:

\section{Proposition 1}

The source encoding of vector $\mathbf{y}_{S-R}$ with side information $\mathbf{y}_{S-D}$ at the decoder can be performed at rates higher than:

$$
r^{*}(\mathbf{d})=\sum_{i=1}^{N_{e i g}} \log \left(\frac{s_{i}}{d_{i}}\right)=\sum_{i=1}^{N_{\text {eig }}} \log \left(1+\frac{s_{i}}{\eta_{i}}\right)
$$

where $d_{i}$ is the distortion on the ith component of $\mathbf{U}^{H} \mathbf{y}_{S-R}$ and satisfies $0 \leq d_{i} \leq s_{i}$ for $1 \leq i \leq N_{\text {eig }}$.

In particular, the rate-distortion function $r^{*}(D)$ is achieved by the well-known reverse-waterfilling algorithm on the eigenvalues of the conditional covariance matrix, i.e.:

$d_{i}=\left\{\begin{array}{l}\lambda \quad \text { if } 0 \leq \lambda<s_{i} \\ s_{i} \quad \text { otherwise }\end{array}\right.$ with $\lambda$ s.t. $\sum_{i=1}^{N_{\text {eig }}} d_{i}=D$
Before proving proposition 1, one can notice that it generalizes some results from rate-distortion theory. For instance, the compression noise introduced by [9] in the Gaussian scalar case is a special case of equation (11) in single dimension. This noise is approximately equal to the distortion when the latter is small, but goes up to infinity when the distortion approaches the source variance. This reflects the fact that a highly distorted signal cannot convey information anymore. Another well-known result (see e.g. [12]) is that the rate-distortion function of parallel Gaussian source is obtained by reverse-waterfilling on the eigenvalues of the source covariance matrix. Here, equation (16) is the same algorithm applied to the eigenvalues of the conditional covariance matrix. The latter reduces to the covariance matrix when the cross-correlation of equation (5) goes to zero or when the SNR at the destination is small. In these two cases, the side information at the destination cannot be exploited to reduce the rate required at the relay to encode its observation with a given distortion.

\section{Proof:}

Following similar notations as [9], we denote by $r^{*}(\mathbf{d})$ the rate with side information at the destination only and by $r_{\mathbf{y}_{S-R} \mid \mathbf{y}_{S-D}}(\mathbf{d})$ the rate with side information at both source and destination. We compute $r_{\mathbf{y}_{S-R} \mid \mathbf{y}_{S-D}}(\mathbf{d})$ by minimizing the mutual information between $\mathbf{y}_{S-R}$ and $\hat{\mathbf{y}}_{S-R}$ w.r.t. their joint distribution $f\left(\mathbf{y}_{S-R}, \hat{\mathbf{y}}_{S-R} \mid \mathbf{y}_{S-D}\right)$ under distortion constraint d. Let first compute this mutual information in the specific case of Fig. 1. From the well-known property of entropy:

$$
H(\mathbf{A x})=H(\mathbf{x})+\log |\mathbf{A}|
$$

we have that:

$$
I\left(\mathbf{y}_{S-R} ; \hat{\mathbf{y}}_{S-R} \mid \mathbf{y}_{S-D}\right)=I\left(\mathbf{U}^{H} \mathbf{y}_{S-R} ; \mathbf{U}^{H} \hat{\mathbf{y}}_{S-R} \mid \mathbf{y}_{S-D}\right)
$$

and from equation (12):

$$
\begin{aligned}
& I\left(\mathbf{y}_{S-R} ; \hat{\mathbf{y}}_{S-R} \mid \mathbf{y}_{S-D}\right) \\
= & H\left(\mathbf{U}^{H} \hat{\mathbf{y}}_{S-R} \mid \mathbf{y}_{S-D}\right)-H\left(\mathbf{U}^{H} \hat{\mathbf{y}}_{S-R} \mid \mathbf{y}_{S-D}, \mathbf{y}_{S-R}\right) \\
= & H\left(\mathbf{A} \mathbf{U}^{H} \mathbf{y}_{S-R}+\mathbf{A} \boldsymbol{\psi}+\mathbf{K} \mathbf{y}_{S-D} \mid \mathbf{y}_{S-D}\right) \\
& -H\left(\mathbf{A} \mathbf{U}^{H} \mathbf{y}_{S-R}+\mathbf{A} \boldsymbol{\psi}+\mathbf{K} \mathbf{y}_{S-D} \mid \mathbf{y}_{S-R}, \mathbf{y}_{S-D}\right)
\end{aligned}
$$

From (17) and the fact that the entropy of a known variable is zero, we can rewrite (18) as:

$$
I\left(\mathbf{y}_{S-R} ; \hat{\mathbf{y}}_{S-R} \mid \mathbf{y}_{S-D}\right)=H\left(\mathbf{U}^{H} \mathbf{y}_{S-R}+\boldsymbol{\psi} \mid \mathbf{y}_{S-D}\right)-H(\boldsymbol{\psi})
$$

As stated in [7], by definition of the CKLT, the components of $\mathbf{U}^{H} \mathbf{y}_{S-R}$ are conditionally independent given $\mathbf{y}_{S-D}$. Therefore, (19) is equivalent to: 


$$
\begin{aligned}
I\left(\mathbf{y}_{S-R} ; \hat{\mathbf{y}}_{S-R} \mid \mathbf{y}_{S-D}\right) & =\sum_{i=1}^{N_{c i g}} \log \left(s_{i}+\frac{s_{i} d_{i}}{s_{i}-d_{i}}\right)-\sum_{i}^{N_{\text {cig }}} \log \left(\frac{s_{i} d_{i}}{s_{i}-d_{i}}\right) \\
& =\sum_{i=1}^{N_{\text {eig }}} \log \left(\frac{s_{i}}{d_{i}}\right)
\end{aligned}
$$

Now for a general distribution $f\left(\mathbf{y}_{S-R}, \hat{\mathbf{y}}_{S-R} \mid \mathbf{y}_{s-D}\right)$, it can be checked that:

$I\left(\mathbf{y}_{S-R} ; \hat{\mathbf{y}}_{S-R} \mid \mathbf{y}_{S-D}\right)=H\left(\mathbf{y}_{S-R} \mid \mathbf{y}_{S-D}\right)-H\left(\mathbf{y}_{S-R} \mid \mathbf{y}_{S-D}, \hat{\mathbf{y}}_{S-R}\right)$

$\geq \log (2 \pi e)^{N_{R}}\left|\mathbf{R}_{y_{S-R} \mid y_{S-D}}\right|-\log (2 \pi e)^{N_{R}}\left|\mathbf{R}_{\mathbf{y}_{S-R}-\hat{\mathbf{y}}_{S-R} \mid y_{S-D}}\right|$

$\geq \sum_{i=1}^{N_{\text {eig }}} \log \left(\frac{s_{i}}{d_{i}}\right)$

Thus, from (20) and (21) we conclude that

$$
r_{\mathbf{y}_{S-R} \mid \mathbf{y}_{S-D}}(\mathbf{d})=\sum_{i}^{N_{\text {eig }}} \log \left(\frac{s_{i}}{d_{i}}\right)
$$

The equality between the rates with and without side information at the source follows from section 3 of [9] and from the equivalence between Fig. 1 and Fig. 2, which leads to:

$$
I\left(\mathbf{y}_{S-R} ; \mathbf{v} \mid \mathbf{y}_{S-D}\right)=I\left(\mathbf{y}_{S-R} ; \hat{\mathbf{y}}_{S-R} \mid \mathbf{y}_{S-D}\right)
$$

where $\mathbf{V}$ is defined on Fig. 2, and in this case:

$$
r^{*}(\mathbf{d})=r_{\mathbf{y}_{S-R} \mid \mathbf{y}_{S-D}}(\mathbf{d})
$$

Finally, the rate-distortion function $r^{*}(D)$ is obtained by minimizing the rate under total squared distortion $D$. This constrained problem is convex and the solution (16) is given by the well-known reverse waterfilling algorithm [12]. This completes the proof.

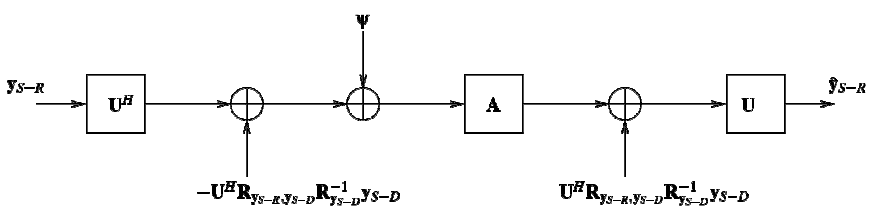

Figure 1: Rate-distortion coding of gaussian vector $\mathbf{y}_{S-R}$ with side information $\mathbf{y}_{S-D}$ at the encoder and decoder

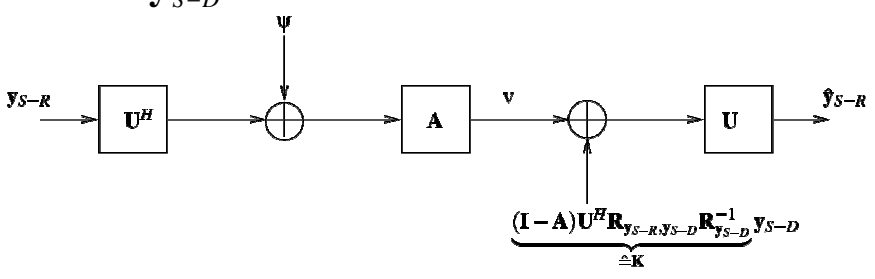

Figure 2: Rate-distortion coding of gaussian vector $\mathbf{y}_{S-R}$ with side

information $\mathbf{y}_{S-D}$ at the decoder

Note that in this paper, we do not address practical implementations of Wyner-Ziv coding. We refer the reader to e.g. [14] for an application of LDPC codes to Wyner-Ziv coding in C\&F relaying on scalar Gaussian channel. Assuming that there exist codes which approach the rate-distortion trade- off of Proposition 1, we now compute the mutual information that can be achieved by a $\mathrm{C} \& \mathrm{~F}$ relay.

\section{ACHIEVABLE C\&F RATES}

In the previous section, we clarified the signal model and the source coding scheme used by the relay. We now investigate how the compression noise introduced by WynerZiv coding affects the mutual information of the cooperative link.

For notational simplicity, we now assume white noise at the relay (i.e. $\mathbf{R}_{\mathbf{n}_{R}}=\sigma_{n, R}^{2} \mathbf{I}_{N_{R}}$ ) and destination. This simplifies the expression of the achievable rate in the proposition below.

\section{Proposition 2}

The following rate is achievable by half-duplex cooperative $C \& F$ relaying on Gaussian vector channels:

$$
I_{C \& F}=\max _{t, \boldsymbol{\eta}} t\left(I_{R}+I\left(\mathbf{x}_{S} ; \mathbf{y}_{S-D}\right)\right)
$$

where:

$$
\begin{gathered}
I_{R}=\sum_{i=1}^{N_{e i g}} \log \left(\frac{s_{i}+\eta_{i}}{\sigma_{n, R}^{2}+\eta_{i}}\right) \\
I\left(\mathbf{x}_{S} ; \mathbf{y}_{S-D}\right)=\log \left|\mathbf{I}_{N_{D}}+\frac{1}{\sigma_{n, D}^{2}} \mathbf{H}_{S-D} \mathbf{R}_{\mathbf{x}_{S}} \mathbf{H}_{S-D}^{H}\right|
\end{gathered}
$$

and the compression noise variance $\boldsymbol{\eta}$ satisfies Proposition 1, i.e.

$$
\sum_{i=1}^{N_{\text {eig }}} \log \left(1+\frac{s_{i}}{\eta_{i}}\right) \leq R(t) \hat{=} \frac{1-t}{t} I\left(\mathbf{x}_{R} ; \mathbf{y}_{D}\right)
$$

As in previous section, we attempt an interpretation of the result before proving it. First it can be checked that in the single dimensional case, equation (25) boils down to HøstMadsen and Zhang's achievable rates given in [6]. The term $t I\left(\mathbf{x}_{S} ; \mathbf{y}_{S-D}\right)$ corresponds to the mutual information that is transmitted by the source directly to the destination during the first slot. The other term $t I_{R}$ corresponds to the additional information about the source that is brought by the compressed observation reconstructed by the destination. It is a sum over the $N_{\text {eig }}$ non-zero eigenmodes of the conditional covariance matrix. In equation (26), one can see that when the compression noise $\eta_{i}$ is negligible compared to the thermal noise variance $\sigma_{n, R}^{2}$ and the "useful signal power" $s_{i}$, then the contribution of this eigenmode to $I_{R}$ is maximized and approximately equal to $\log \left(s_{i} / \sigma_{n, R}^{2}\right)$. Of course when the compression noise is high, then the eigenmode cannot bring information. The highest achievable $\mathrm{C} \& \mathrm{~F}$ rate is obtained not only by maximizing (25) over the time sharing $t$, but also by finding the optimum compression noise variance vector $\boldsymbol{\eta}$ that 
maximizes $I_{R}$ for a given value of $t$. The solution to this second optimization problem is given by proposition 3 and leads to a solution that differs from the reverse-waterfilling of equation (16).

\section{Proof:}

Let first notice that the last term $\mathbf{U K} \mathbf{y}_{S-D}$ of (12) does not affect the mutual information:

$I\left(\mathbf{x}_{S} ; \hat{\mathbf{y}}_{S-R}, \mathbf{y}_{S-D}\right)=I\left(\mathbf{x}_{S} ; \mathbf{U A U} \mathbf{U}^{H} \mathbf{y}_{S-R}+\mathbf{U A} \boldsymbol{\psi}, \mathbf{y}_{S-D}\right)$

Moreover, from property (17), the product by matrix $\mathbf{U A}^{-1} \mathbf{U}^{H}$ does not affect mutual information either:

$$
I\left(\mathbf{x}_{S} ; \hat{\mathbf{y}}_{S-R}, \mathbf{y}_{S-D}\right)=I\left(\mathbf{x}_{S} ; \mathbf{y}_{S-R}+\mathbf{U} \boldsymbol{\psi}, \mathbf{y}_{S-D}\right)
$$

Therefore, defining

$$
\tilde{\mathbf{y}}_{S-R} \hat{=} \mathbf{y}_{S-R}+\mathbf{U} \boldsymbol{\psi}
$$

We have that:

$$
I\left(\mathbf{x}_{S} ; \hat{\mathbf{y}}_{S-R}, \mathbf{y}_{S-D}\right)=I\left(\mathbf{x}_{S} ; \tilde{\mathbf{y}}_{S-R}, \mathbf{y}_{S-D}\right)
$$

The mutual information of the cooperative link is therefore that of a virtual $N_{S} \times\left(N_{R}+N_{D}\right)$ MIMO channel where the signal received at the $N_{R}$ "remote antennas" undergoes an additional compression noise as shown by (30). In order to prove proposition 2, we need to further simplify (31). From the chain rule we have:

$$
I\left(\mathbf{x}_{S} ; \tilde{\mathbf{y}}_{S-R}, \mathbf{y}_{S-D}\right)=I\left(\mathbf{x}_{S} ; \mathbf{y}_{S-D}\right)+\underbrace{I\left(\mathbf{x}_{S} ; \tilde{\mathbf{y}}_{S-R} \mid \mathbf{y}_{S-D}\right)}_{\triangleq I_{R}}
$$

While the computation of $I\left(\mathbf{x}_{S} ; \mathbf{y}_{S-D}\right)$ is straightforward, it turns out that a simple closed form expression can also be found for $I_{R}$. From the definition of mutual information:

$$
I_{R}=H\left(\mathbf{y}_{S-R}+\mathbf{U} \boldsymbol{\psi} \mid \mathbf{y}_{S-D}\right)-H\left(\mathbf{y}_{S-R}+\mathbf{U} \boldsymbol{\psi} \mid \mathbf{x}_{S, \mathbf{y}_{S-D}}\right)
$$

Since $\mathbf{y}_{S-D}$ is a noisy version of $\mathbf{H}_{S-D} \mathbf{x}_{S}$, (33) simplifies as:

$$
\begin{aligned}
& I_{R}=H\left(\mathbf{y}_{S-R}+\mathbf{U} \psi \mid \mathbf{y}_{S-D}\right)-H\left(\mathbf{y}_{S-R}+\mathbf{U} \boldsymbol{\psi} \mid \mathbf{x}_{S}\right)
\end{aligned}
$$

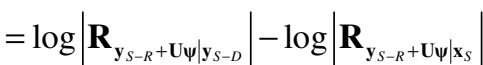

$$
\begin{aligned}
& =\log \left|\mathbf{R}_{\boldsymbol{y}_{S-R} \mid \mathbf{y}_{S-D}}+\mathbf{U d i a g}(\boldsymbol{\eta}) \mathbf{U}^{H}\right|-\log \left|\mathbf{R}_{\mathbf{y}_{S-R} \mid \mathbf{s}_{S}}+\mathbf{U d i a g}(\boldsymbol{\eta}) \mathbf{U}^{H}\right| \\
& =\log \left|\mathbf{U d i a g}\left(s_{i}+\eta_{i}\right)_{1 \leq i \leq N_{\text {cig }}} \mathbf{U}^{H}\right|-\log \mid \mathbf{U d i a g}\left(\sigma_{n, R}^{2}+\eta_{i}\right)_{1 \leq i \leq N_{\text {cig }}} \mathbf{U}^{H} \\
& =\sum_{i=1}^{N_{e i g}} \log \left(\frac{s_{i}+\eta_{i}}{\sigma_{n, R}^{2}+\eta_{i}}\right)
\end{aligned}
$$

This concludes the proof of proposition 2 .

Now that a set of C\&F achievable rates has been derived, we are interested in the maximum rate within this set. It can be found by solving the following optimization problem:

$$
\begin{aligned}
& \max _{\boldsymbol{\eta}} \sum_{i=1}^{N_{\text {eig }}} \log \left(\frac{s_{i}+\eta_{i}}{\sigma_{n, R}^{2}+\eta_{i}}\right) \\
& \text { s.t. }\left\{\begin{array}{l}
\sum_{i=1}^{N_{\text {eig }}} \log \left(1+\frac{s_{i}}{\eta_{i}}\right) \leq R(t) \\
\eta_{i} \geq 0 \text { for } 1 \leq i \leq N_{\text {eig }}
\end{array}\right.
\end{aligned}
$$

Unfortunately, (35) is not a convex minimization problem in standard form, because although it can be checked that the constraints and objective are convex in $\boldsymbol{\eta}$, the objective has to be maximized. Therefore, we introduce a change of optimization variable, by defining

$$
r_{i} \hat{=} \log \left(1+\frac{s_{i}}{\eta_{i}}\right)
$$

The variable $r_{i}$ corresponds to the rate selected for the quantization of the $i$ th component of the signal at the output of the CKLT.

Inserting (36) into (35) leads to:

$$
\begin{aligned}
& \max _{\mathbf{r}} \sum_{i=1}^{N_{\text {eig }}} \log \left(\frac{s_{i} 2^{r_{i}}}{\sigma_{n, R}^{2}\left(2^{r_{i}}-1\right)+s_{i}}\right) \\
& \text { s.t. }\left\{\begin{array}{l}
\sum_{i=1}^{N_{\text {eig }}} r_{i} \leq R(t) \\
r_{i} \geq 0 \text { for } 1 \leq i \leq N_{\text {eig }}
\end{array}\right.
\end{aligned}
$$

This time, it can be checked that the objective is concave in $\mathbf{r}$. Therefore, the problem (37) is convex and can be solved by writing the KKT conditions. This leads, after a few simple calculations, to the following proposition:

\section{Proposition 3}

Among the set of achievable $C \& F$ rates defined by proposition 2, the highest rate is obtained when

$$
\begin{gathered}
\eta_{i}=\frac{s_{i}}{2^{r_{i}}-1} \\
r_{i}=\left[\mu+\log \left(\frac{s_{i}}{\sigma_{n, R}^{2}}-1\right)\right]^{+}
\end{gathered}
$$

where

and $\mu$ is a constant such that the rate constraint is reached:

$$
\sum_{i=1}^{N_{e i g}} r_{i} \leq R(t)
$$

We now try to highlight the differences between proposition 3 and reverse-waterfilling of the distortion. In reverse waterfilling, the algorithm tries to spread the distortion uniformly, under the constraint that $d_{i} \leq s_{i}$. Such a strategy would lead to $r_{i}=\log \left(s_{i} / d\right)$ with $d$ a constant corresponding to the distortion on the eigenmodes which are 
worth quantizing (i.e. $r_{i}>0$ ). Now the solution in proposition 3 has a rate water-filling interpretation. The ratio $s_{i} / \sigma_{n, R}^{2}$ can be interpreted as a signal to thermal noise ratio per eigenmode. It can be checked, by definition of the CKLT, that this ratio is always greater than 1. From (26), we see that the eigenmodes which have a high $s_{i} / \sigma_{n, R}^{2}$ ratio are the largest contributors to the $\mathrm{C} \& \mathrm{~F}$ mutual information provided their compression noise is low-enough. The term $\log \left(\frac{s_{i}}{\sigma_{n, R}^{2}}-1\right)$ in equation (39) can thus be interpreted as a rate penalty for the eigenmodes which have a lower potential contribution to $\mathrm{C} \& \mathrm{~F}$ mutual information. The penalty tends to $-\infty$ when $s_{i} / \sigma_{n, R}^{2}$ tends to 1 , and in this case the eigenmode will not be quantized. In other words, the rate water-filling algorithm grants more bits (or equivalently less distortion) to eigenmodes which have a larger contribution to $\mathrm{C} \& \mathrm{~F}$ mutual information.

Finally, to come back to our simplifying assumption of orthogonal source and relay transmissions during the second slot, notice that if this constraint is relaxed, then $S$ can send a new independent message at a rate constrained to lie within the MAC capacity region. The latter can be achieved by successive decoding. If the relayed message is decoded first, then the source signal during the $2^{\text {nd }}$ slot shall be treated as noise, and equation (6) shall be modified accordingly.

\section{SIMULATION RESULTS}

In the previous section, we derived achievable $C \& F$ rates and showed that the conventional source coding strategy which minimizes total distortion is outperformed by the strategy which maximizes the $\mathrm{C} \& \mathrm{~F}$ mutual information. In order to assess the performance improvement, we simulate the achievable bit rate in the same simulation scenario as in [8], which corresponds to the uplink of a cellular system similar to IEEE802.16. We consider a Base Station (BS) and a fixed Relay Station (RS), both equipped with 4 antennas. They are assumed in Line-Of-Sight propagation conditions, with a very good link budget $\left(S N R_{R S-B S}=30 \mathrm{~dB}\right.$ ). The Mobile Station (MS) has 2 antennas and is in Non-Line Of Sight situation from both the BS and RS, with a bad link budget to the BS $\left(S N R_{M T-B S}=0 \mathrm{~dB}\right)$. We assume a MIMO-OFDM physical layer with $10 \mathrm{MHz}$ channel spacing, an FFT size of 256, a number of useful subcarriers equal to 192 and a cyclic prefix length representing $1 / 8$ of the useful symbol. The propagation conditions correspond to an urban micro-cell channel model valid between $2 \mathrm{GHz}$ and $5 \mathrm{GHz}$, as described in [13]. We also assume isotropic transmission at the Source and Relay. Although the optimization of transmit covariance matrices is an interesting problem, we prefer to defer it to a future paper for lack of space. On Figure 3, we plot the ergodic achievable rate with various transmission strategies when the quality of the MT to RS link ( $S N R_{M T-R S}$ ) varies from $-5 \mathrm{~dB}$ to $10 \mathrm{~dB}$. As expected and already observed in e.g. [2], C\&F always outperforms direct link and also A\&F because time sharing parameter can be optimized. Moreover, $\mathrm{C} \& \mathrm{~F}$ is outperformed by $\mathrm{D} \& \mathrm{~F}$ when the SNR on the S-R link exceeds a certain threshold (here, around $4 \mathrm{~dB}$ ). The two dashed curves correspond to the minimum distortion and maximum mutual information $C \& F$ strategies. It can be seen that the performance difference between the two strategies is not very large. This can be explained by the fact that reversewaterfilling, although not optimum, is not a bad strategy since it also tends to grant more bits to the eigenmodes associated to the largest eigenvalues $S_{i}$. Finally, the solid black curve is an upper-bound corresponding to infinite R-D link capacity, allowing virtual MIMO without any compression noise. The significant gap between this upper-bound and the C\&F achievable rates highlights the need to increase the RS to BS link capacity by deploying for instance dedicated highcapacity directional links between the RS and BS in order to fully exploit $\mathrm{C} \& \mathrm{~F}$ relaying in real systems.

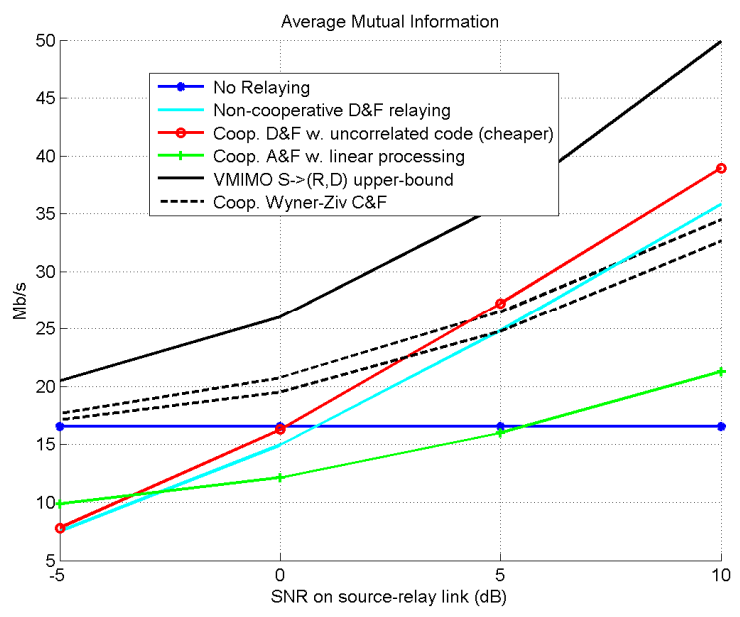

Figure 3: Achievable rates with cooperative C\&F relaying in a MIMOOFDM WMAN uplink scenario compared to alternative transmission strategies

\section{CONCLUSION}

We have derived in this paper a $\mathrm{C} \& \mathrm{~F}$ coding strategy which maximizes the achievable rates on Gaussian vector channels, thus extending previous results valid for Gaussian scalar channel. We have analyzed the specificity of this new strategy compared to the conventional source-coding approach which minimizes total squared distortion, and evaluated its performance by simulations in a practical scenario. Several extensions of the results published in this paper could be topics for further investigation, for instance the extension to multiple relays.

\section{ACKNOWLEDGMENT}

This work was performed in the framework of the IST FIREWORKS project, which is funded by the European Commission. 


\section{REFERENCES}

[1] K. Gosse, M. de Courville, M. Muck, S. Rouquette and S. Simoens Chapter 22: MIMO wireless local area networks In "Space-Time Wireless Systems, From Array Processing to MIMO Communications" Edited by H. Bölcskei, D. Gesbert, C. B. Papadias and A.-J. van der Veen, Cambridge University Press, 2006

[2] G. Kramer, M. Gastpar, P. Gupta, "Cooperative Strategies and Capacity Theorems for Relay Networks", IEEE Trans. on Information Theory, Vol 51. No 9. Sep 2005

[3] Nosratinia, T. E. Hunter, A. Hedayat, "Cooperative Communication in Wireless Networks", IEEE Communications Magazine, Oct 2004

[4] O. Muñoz, J. Vidal, A. Agustin, "Non-Regenerative MIMO Relaying with Channel State Information", in Proc. IEEE ICASSP, 2005

[5] N. Varanese, O. Simeone, Y. Bar-Ness, U. Spagnolini “Achievable Rates of Multi-Hop and Cooperative Amplify-and-Forward Relay Systems with Full CSI" Proceedings of IEEE SPAWC, Jul 2006

[6] A. Høst-Madsen, J. Zhang "Capacity Bounds and Power Allocation for Wireless Relay channels" IEEE Transactions on Information Theory, Vol 51, N6, pp. 2020-2040, June 2005

[7] M. Gastpar, P.L. Dragotti and M. Vetterli "On compression using the distributed Karhunen Loeve Transform” Proceedings of IEEE ICASSP, May 2004

[8] S. Simoens, J. Vidal, O. Muñoz "Compress-and-Forward cooperative relaying in MIMO-OFDM systems" Proceedings of IEEE SPAWC, July 2006

[9] A.D. Wyner "The rate-distortion function for source coding with side information at the decoder -II: General Sources" Information and Control, Vol 38, pp 60-80, 1978

[10] T. M. Cover and A.A. El Gamal, "Capacity theorems for the relay channel", IEEE Transactions on Information Theory, vol. IT-25, No 5 , pp 572-584, Sep 1979

[11] T.K. Moon, W.C. Stirling "Mathematical Methods and Algorithms for Signal Processing" Prentice Hall, 2000

[12] T. Cover, J.A. Thomas "Elements of Information Theory" WileyInterscience, 1991

[13] D.S. Baum et. al., "An Interim Channel Model for Beyond-3G Systems" in Proc. IEEE VTC Spring, Vol 5, May 2005.

[14] Zhixin Liu, Vladimir Stankovic and Zixiang Xiong "Wyner-Ziv coding for the half-duplex relay channel" Proceedings of IEEE ICASSP, March 2005

[15] E. Telatar, "Capacity of multi-antenna Gaussian channels" European Trans. Telecommun., vol. 10, pp. 585--595, 1999

[16] Agustin, O. Munoz, J. Vidal, D. Perez, G. Vasquez "Medium Access, Resource Allocation and Capacity Evaluation of Cooperative MIMO Systems" IST-2001-32549 ROMANTIK Project D4.4.1 